\title{
Diagnostische und therapeutische Aspekte bei Wirbelsäulenkrankheiten
}

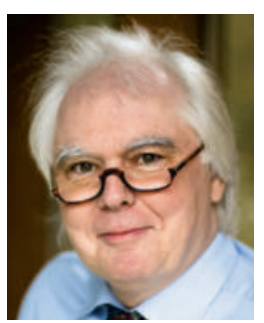

Prof. Dr. Wolfgang Rüther
Sehr verehrte Kolleginnen, sehr geehrte Kollegen,

in der vorliegenden Ausgabe präsentieren wir Ihnen 5 besonders interessante Publikationen, die in den letzten Monaten in deutschsprachigen Zeitschriften erschienen sind. Die Arbeiten beschäftigen sich mit diagnostischen und therapeutischen Aspekten bei Wirbelsäulenkrankheiten. Bei der Auswahl bin ich versucht, operative von nicht operativen Themen zu trennen. Meines Erachtens kennzeichnet diese Versuchung die aktuelle Diskussion um die Wirbelsäulenkrankheiten. Die Operation ist eine von vielen therapeutischen Optionen in der Vertebrologie - nicht mehr und nicht weniger; aber die Operationen und ihre Details scheinen aktuell die Literatur geradezu zu prägen. Ist das nicht eine Schieflage?

Die Arbeit von A. Mehrkens betont noch einmal mehr, dass die Operation an der Wirbelsäule nicht die letzte und ultimative therapeutische Option darstellt, wenn trotz aller therapeutischen Bemühungen der Schmerz persistiert. Dieser gedankliche Ansatz mag in der Endoprothesenchirurgie eine gewisse Berechtigung haben, indem der endoprothetische Gelenkersatz am Ende der therapeutischen Eskalation steht. Der Autor unterstreicht und begründet, dass die Operation an der Wirbelsäule in präzisen Indikationen ihren erfolgreichen Einsatz findet - und nicht im therapeutischen Endpunkt nach nicht ausreichend erfolgreicher Schmerztherapie.

Die Statik der Wirbelsäule nicht nur in frontaler, sondern vor allem in der sagittalen Ebene steht im Mittelpunkt der Publikation von C. Thomé. Die Problematik ist seit Jahren bekannt. Die rekonstruktive Wirbelsäulenchirurgie strebt das ausgeglichene sagittale Profil der Wirbelsäule an - nicht immer mit Erfolg - und am Ende persistiert dann eine schmerzhafte Wirbelsäule. Die Arbeit macht auf das Problem aufmerksam; es verlangt eine spezielle Diagnostik, nicht nur postoperativ, sondern auch bei der konservativen Therapie und präoperativ. Wichtig: daran denken!

Die Injektionstherapie an der Wirbelsäule gehört seit Jahrzehnten zum Repertoire interventioneller Therapie an der Wirbelsäule. Die Arbeit von F. Jablawi und K. Schöller konzentriert sich auf Injektionen mit Kortison. Sie reflektiert den aktuellen Wissensstand und beschreibt die aktuellen theoretischen Hintergründe. Die Autoren erinnern nachdrücklich daran, dass verschiedene Präparate, an der Wirbelsäule verwendet, als Off-Label-Use zu betrachten sind! „Die wissenschaftliche Evidenz zur Effektivität der verschiedenen wirbelsäulennahen Steroidinfiltrationen steht in einem starken Kontrast zu deren häufigen Anwendungen." Das darf man als eine Art Weckruf, wenn nicht gar Warnung, verstehen.

Die klinischen Bilder entzündlich rheumatischer Gelenkkrankheiten haben sich in den letzten Jahren gewandelt - u. a. infolge effektiver medikamentöser Behandlungsregimes. Das gilt auch für den kraniozervikalen Übergang; seine entzündlich rheumatische Instabilität wird seltener gesehen - und es wird auch seltener darüber berichtet. R. Kothe ist einer der Experten für dieses Feld. Er gibt einen Überblick über die aktuellen Standards.

Die Publikation von K. S. C. Gollisch et al. verrät schon im Titel ihre Intention: ein spannender Fall, der zu denken gibt.

Ich wünsche Ihnen, unseren geneigten Lesern, eine anregende Lektüre mit klaren „take home messages" für Ihren klinischen Alltag.

Herzlichst

Ihr

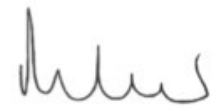

